\title{
Plasmonic Dirac Cone in Twisted Bilayer Graphene
}

\author{
Luis Brey and T. Stauber \\ Materials Science Factory, Instituto de Ciencia de Materiales de Madrid (CSIC), Cantoblanco, 28049 Madrid, Spain \\ T. Slipchenko and L. Martín-Moreno® \\ Instituto de Nanociencia y Materiales de Aragón (INMA), CSIC-Universidad de Zaragoza, Zaragoza 50009, Spain \\ and Departamento de Física de la Materia Condensada, Universidad de Zaragoza, Zaragoza 50009, Spain
}

(Received 21 June 2020; accepted 16 November 2020; published 18 December 2020)

\begin{abstract}
We discuss plasmons of biased twisted bilayer graphene when the Fermi level lies inside the gap. The collective excitations are a network of chiral edge plasmons (CEP) entirely composed of excitations in the topological electronic edge states that appear at the AB-BA interfaces. The CEP form a hexagonal network with a unique energy scale $\epsilon_{p}=\left(e^{2}\right) /\left(\epsilon_{0} \epsilon t_{0}\right)$ with $t_{0}$ the moiré lattice constant and $\epsilon$ the dielectric constant. From the dielectric matrix we obtain the plasmon spectra that has two main characteristics: (i) a diverging density of states at zero energy, and (ii) the presence of a plasmonic Dirac cone at $\hbar \omega \sim \epsilon_{p} / 2$ with sound velocity $v_{D}=0.0075 c$, which is formed by zigzag and armchair current oscillations. A network model reveals that the antisymmetry of the plasmon bands implies that CEP scatter at the hexagon vertices maximally in the deflected chiral outgoing directions, with a current ratio of 4/9 into each of the deflected directions and 1/9 into the forward one. We show that scanning near-field microscopy should be able to observe the predicted plasmonic Dirac cone and its broken symmetry phases.
\end{abstract}

DOI: 10.1103/PhysRevLett.125.256804

Introduction.-The study of graphene has brought to light many unexpected basic as well as applied physical properties [1,2]. More surprises appear when two graphene layers are stacked and rotated one on top of the other, forming the so-called twisted bilayer graphene (TBG). When the rotation angle is large, the graphene layers are electronically decoupled [3,4], but at small twist angles the Fermi velocity of the carriers reduces considerably [5]. At some particular magic angle, the electronic bands become almost flat around charge neutrality [6-8]. In this regime, new and unpredicted electronic phases emerge [9-12].

Also collective modes in twisted structures have been studied and show several new features [13-23]. In this Letter, however, we will discuss the collective excitations associated with the network of chiral electronic edge states (EES) that appear when an electric field is applied perpendicular to the sample and the chemical potential lies inside the gap [24-26]. In this regime, plasmons can be described by a simple macroscopic model that is defined by a hexagonal lattice of alternating Hall conductivities, as shown in Fig. 1(d).

The main results of our work are (i) that plasmons are well represented by chiral edge plasmons (CEP) bounded to the AB-BA interfaces, that scatter at the hexagon vertices mainly in the two deflected chiral outgoing directions and (ii) the prediction of a plasmonic Dirac cone, which is formed by spinors composed of oscillating current patterns in the zigzag and armchair direction, respectively. A plasmonic gap can be opened by breaking the rotational symmetry via strain or due to magnetic fields. Furthermore, the plasmonic density of states diverges in the limit of vanishing frequency. We propose that these features can be seen in scanning near-field microscopy.

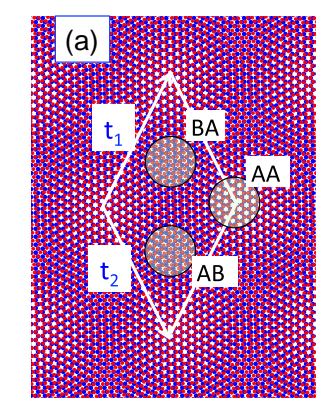

(b)

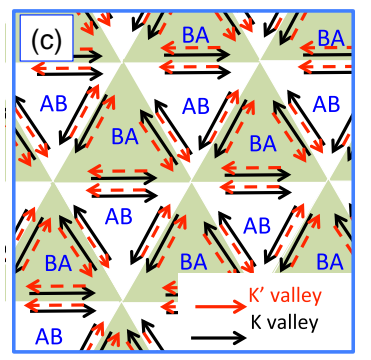

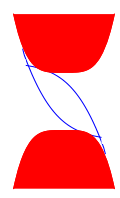

$K^{\prime}$ valley

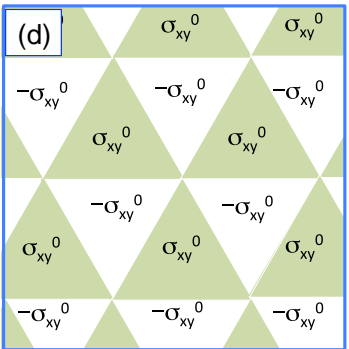

FIG. 1. (a) TBG's unit cell showing the regions with stacking $\mathrm{AA}, \mathrm{AB}$, and BA. (b) Propagating and antipropagating edge modes corresponding at the $K$ and $K^{\prime}$ valleys, respectively, at gapped $\mathrm{AB} / \mathrm{BA}$ interfaces. (c) Hexagonal pattern showing unit cell with stacking $\mathrm{AB}$ and $\mathrm{BA}$ and the network of EES encircling these regions. (d) Hexagonal pattern showing the periodicity of triangular regions with Hall conductivities $\pm \sigma_{x y}^{0}$. 
Model.-The TBG forms a moiré pattern that, although in general is incommensurate, can be approximated very accurately by a triangular unit cell of lattice parameters $\mathbf{t}_{1(2)}=[(1 / 2),( \pm \sqrt{3} / 2)] t_{0}$, with $t_{0}=(a) /(2 \sin \theta / 2)$, being $a=2.46 \AA$ the graphene lattice parameters and $\theta$ the twist angle; see Fig. 1(a). In the TBG unit cell, it is possible to identify regions where the graphene layers are either Bernal-stacked (AB and $\mathrm{BA}$ ) or one on top of the other (AA); see Fig. 1(a). An interlayer bias, $\Delta$, opens an electronic gap in the Bernal stacked regions [27]. Under lattice relaxation, these regions expand at the expense of the AA regions in order to reduce elastic energy [28]. For small twist angles, the Bernal-stacked regions define a periodic hexagonal structure, with a unit cell formed by two triangles with stacking $\mathrm{BA}$ and $\mathrm{AB}$, respectively; see Fig. 1(c). This regime has recently attracted considerable attention by various research groups [29,30].

Gapped Bernal-stacked regions are nontrivial insulators and, consequently, have a finite valley Chern number per spin [25,31-33], i.e., $C_{K}^{\mathrm{AB}}=-C_{K^{\prime}}^{\mathrm{AB}}=-C_{K}^{\mathrm{BA}}=C_{K^{\prime}}^{\mathrm{BA}}=\operatorname{sgn}(\Delta)$, where $K$ and $K^{\prime}$ are the graphene Dirac points. At the interface between the $\mathrm{AB}$ and $\mathrm{BA}$ regions, and because of the difference in band topology, two EES per spin and valley appear [26,34,35], with opposite propagation direction in opposite valleys, which has been experimentally proven [36-40]. In TBGs the existence of a network of EES encircling the triangular regions with $\mathrm{AB}$ and $\mathrm{BA}$ stacking has been predicted [41-46] and recently experimentally observed [47].

When the Fermi edge is inside the gapped regions, the plasmonic collective excitations are formed by electronhole electronic transitions in the EES and have an onedimensional chiral character [48]. We name them chiral edge plasmons. In the context of the quantum Hall effect [49-52], CEP exist at the frontier of two-dimensional electron gases with Hall conductivities $C_{1}\left(e^{2} / h\right)$ and $C_{2}\left(e^{2} / h\right)$, and have a dispersion of the form $\hbar \omega_{1 d}(\mathbf{q}) \approx$ $\left(e^{2} / 2 \epsilon \epsilon_{0}\right) q \log (q \delta)\left(C_{1}-C_{2}\right)$, with $\delta$ the effective thickness of the two-dimensional system. A similar dispersion has been obtained for CEPs at the interface between topological insulators with different Chern numbers $[53,54]$. In TBG, we expect that because of the Hall conductivity patches, Fig. 1(d), the collective excitations will consist of a network of CEP.

Formalism.-In order to discuss the emerging plasmonic modes, we use the approach based on the current rather than the charge response [49]. The information on the system is given by the conductivity tensor, which in our case has only nondiagonal Hall components with values $\pm \sigma_{x y}^{0}=g_{s}\left(e^{2} / h\right)$ in the upper and lower triangles of the moiré superlattice; see Fig. 1(d). Here $g_{s}=2$ is the spin degeneracy. For a wave vector $\mathbf{q}$ in the moiré Brillouin zone (MBZ), the frequencies of the dielectric modes $[55,56]$ are obtained from the eigenvalues of the matrix

$$
M_{\mathbf{G}, \mathbf{G}^{\prime}}(\mathbf{q})=-\frac{i}{S_{u c}} \frac{1}{2 \epsilon_{0} \epsilon} \frac{e^{-\frac{\delta}{2}\left(|\mathbf{q}+\mathbf{G}|+\left|\mathbf{q}+\mathbf{G}^{\prime}\right|\right)}}{\sqrt{|\mathbf{q}+\mathbf{G}|\left|\mathbf{q}+\mathbf{G}^{\prime}\right|}}\left[\hat{z} \cdot(\mathbf{q}+\mathbf{G}) \times\left(\mathbf{q}+\mathbf{G}^{\prime}\right)\right] \tilde{\sigma}_{x y}\left(\mathbf{G}-\mathbf{G}^{\prime}\right),
$$

where $S_{u c}$ and $\mathbf{G}$ are the unit cell area and the reciprocal vectors of the moiré lattice, respectively, $\tilde{\sigma}_{x y}$ is the Fourier transform of the Hall conductivity, and $e^{-q \delta} /\left(2 \epsilon_{0} \epsilon q\right)$ is the Fourier transform of the Coulomb interaction in two dimensions. For a given eigenvalue $\omega_{i}(\mathbf{q})$, with eigenvector $\left\{\alpha_{\mathbf{q}}^{i}(\mathbf{G})\right\}$, the corresponding dielectric eigenmode has the form, $\phi^{i}(\mathbf{q}, \mathbf{G})=\left[\left(e^{-\frac{\delta}{2}|\mathbf{q}+\mathbf{G}|}\right) /(\sqrt{|\mathbf{q}+\mathbf{G}|})\right] \alpha_{\mathbf{q}}^{i}(\mathbf{G})$. We have checked that this formalism gives the correct chiral xedge plasmons when the Hall conductivity is modulated only in one direction. For details, see the Supplemental Material [57].

The matrix $M_{\mathbf{G}, \mathbf{G}^{\prime}}(\mathbf{q})$ is real and symmetric, and therefore its eigenvalues and eigenvectors are real. The spectrum is antisymmetric with respect to zero frequency and zero momentum, i.e., for each eigenvalue $\omega(\mathbf{q})$, there exists a corresponding eigenvalue $\omega(-\mathbf{q})=-\omega(\mathbf{q})$ with the same eigenvector. This symmetry in reciprocal frequency-momentum space directly arises from the existence of real valued time-dependent electric fields [54,61]. Because of the underlying lattice symmetry, we also have $\omega(\mathbf{q})=\omega\left(-q_{x}, q_{y}\right)=-\omega\left(q_{x},-q_{y}\right)=\omega\left(R_{\frac{2 \pi}{3}} \mathbf{q}\right)=-\omega\left(R_{\frac{\pi}{3}} \mathbf{q}\right)$.
Plasmon dispersion.-Collective charge density excitations of gapped twisted bilayer graphene are given by the positive eigenvalues of the matrix $M_{\mathbf{G}, \mathbf{G}^{\prime}}(\mathbf{q})$. Because of the chiral nature of the excitations they only exist in half of the MBZ, see shadow regions in Fig. 2(a). The complementary white regions are occupied by plasmonic excitations belonging to the opposite graphene valley. At low energies, the collective excitations are CEP moving along the AB-BA boundaries with a chiral sense of rotation imposed by the arrangement of the Hall conductivity. The character of the excitations becomes clear when we plot the electrical current associated with a dielectric mode, $\vec{j}^{i}(\mathbf{r})=-\sigma_{x y}(\mathbf{r}) \vec{\nabla} \phi^{i}(\mathbf{q}, \mathbf{r})$. In Figs. 3(a) and 3(b), we plot the $x$ and $y$ components, respectively, of the electrical current for a mode near $\Gamma$. The current is localized at the sides of the triangles that form the unit cell and circulates in opposite directions in triangles with opposite Hall conductivity.

In Fig. 2(d), we plot the first nonzero eigenvalues of $M_{\mathbf{G}, \mathbf{G}^{\prime}}(\mathbf{q})$ along the direction $\Gamma-\mathcal{K}^{\prime}-M-\mathcal{K}-\Gamma$ of the MBZ. [62] The most striking feature of the network of CEP 

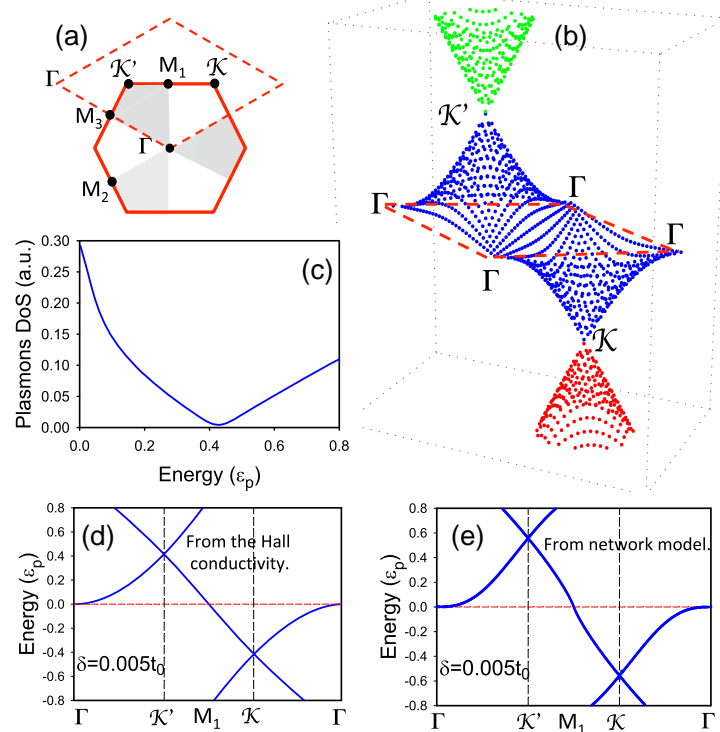

FIG. 2. (a) Moiré Brillouin zone. Plasmons exit in the shadow regions. (b) Dispersion of the dielectric modes in the MBZ. Dirac cones appearing at the points $\mathcal{K}$ and $\mathcal{K}^{\prime}$. Red dashed line shows the plane of zero energy. (c) Plasmonic density of states. The flat dispersion in the $\Gamma-M-\Gamma$ direction produce a high density of states at low energies. The DOS decays to zero at the frequency of the vertex of the plasmonic Dirac cone. (d) Dispersion of the dielectric modes along the direction $\Gamma-\mathcal{K}^{\prime}-M-\mathcal{K}-\Gamma$ of the MBZ. (e) As in (d) but obtained with a network model of CEP that scatter at vertices.

dispersion is the existence of a Dirac point at the $\mathcal{K}^{\prime}$ point of the MBZ. The dielectric modes for momentum $\mathbf{q}$, near the $\mathcal{K}^{\prime}$ point, $\mathbf{q}=\mathcal{K}^{\prime}+\mathbf{k}$, can be written in terms of spinors $\psi_{+}=\left(\begin{array}{c}\sin \theta_{\mathbf{k}} / 2 \\ \cos \theta_{\mathbf{k}} / 2\end{array}\right) e^{i \mathbf{k r}}$ and $\psi_{-}=\left(\begin{array}{c}\cos \theta_{\mathbf{k}} / 2 \\ -\sin \theta_{\mathbf{k}} / 2\end{array}\right) e^{i \mathbf{k r}}$ with $\theta_{\mathbf{k}}=\tan ^{-1}\left(k_{y} / k_{x}\right)$. The up and down spinors are the dielectric modes of the degenerated CEP at the point $\mathcal{K}^{\prime}$. Therefore, near $\mathcal{K}^{\prime}$ the CEP are described by the rotated Dirac equation $H=\hbar v_{D}\left(\sigma_{z} k_{x}+\sigma_{x} k_{y}\right)+E_{D}$, being $v_{D}$ and $E_{D}$ the velocity of the plasmons near $\mathcal{K}^{\prime}$ and the energy of the plasmons at the vertex of the cone, respectively, see Fig. 2(c). Figure 3(d) shows schematically the electrical currents of the CEP at the $\mathcal{K}^{\prime}$ point; they correspond to currents moving along the sides of the triangular networks in the $\hat{x}$ (armchair) and $\hat{y}$ (zigzag) directions. The actual form of the current, as obtained numerically, is plotted in the Supplemental Material [57].

In Fig. 2(c), we plot the plasmons density of states (DOS) as function of frequency. The linear dispersion near the Dirac point $\mathcal{K}^{\prime}$ leads to a vanishing DOS at the energy of the Dirac cone vertex $E_{D}$. On the other hand, the $\omega(-\mathbf{q})=-\omega(\mathbf{q})$ symmetry, combined with the chiral character of the plasmons, produces zero energy excitations along the zone boundaries of half the MBZ, see Figs. 2(a)-2(b) and a peak in the plasmon DOS at zero frequency; see Fig. 2(c).
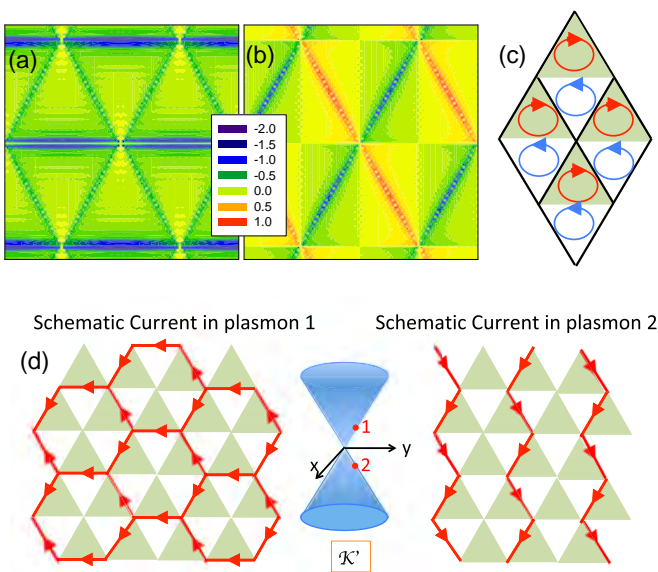

FIG. 3. Currents in the (a) $x$ and (b) $y$ directions for the low energy plasmons near the $\Gamma$ point of the MBZ. (c) Schematic picture of the edge currents corresponding to this plasmon. Schematic picture of the currents corresponding to the two plasmons at the Dirac point $\mathcal{K}^{\prime}$. The plasmons bring electrical currents in perpendicular directions.

Plasmons in TBG as a network of CEP.-The currents depicted in Fig. 3 suggest that plasmons in TBG may be described by a network model [63], similar to that used to describe the low-energy dispersion relation of the electronic system $[43,64]$. We thus expect only weak dependence on the carrier density in contrast to its electronic counterpart [44].

The plasmonic network is composed by the CEP of the AB-BA edges and vertices, where the incoming edge plasmon is scattered in the three possible outgoing directions, see Fig. 1(c). This model incorporates Coulomb interactions through the CEP dispersion relation, $\omega_{1 d}(\mathbf{q})$, but neglects the interedge interactions. Symmetry and current conservation impose that the scattering by a vertex is defined by just two parameters: (i) the ratio between the transmitted current flowing in the forward $P_{f}$, and each deflected direction $P_{d}$, and (ii) the phase of the outgoing forward plasmon, $\phi$. Remarkably, our results [57] show that the antisymmetry of the plasmon spectrum alone fixes the values $P_{f}=1 / 9$ and $P_{d}=4 / 9$, and forces $\phi$ to be either 0 or $\pi$. The parabolicity of the spectrum at the $\Gamma$ point fixes $\phi=\pi$. See Figs. 2(d) and 2(e) for a comparison between the plasmon dispersion and the one provided by the network model.

Symmetry breaking.-The existence of a Dirac point indicates the possibility of opening a gap in the CEP spectrum by, e.g., privileging clockwise electric currents of the form $J_{x}+i J_{y}$ over anticlockwise currents $J_{x}-i J_{y}$. This can be achieved by applying a magnetic field perpendicularly to the layers. In that case, an energy gap will appear near $E_{D}$ and in the presence of a magnetic field, biased TBG will thus become a photonic crystal for nanolight. Also elastic strain should open up a gap by breaking the rotational symmetry. Arguably, the most 
interesting prospect would be the possibility of creating a Haldane gap leading to topologically protected plasmonic currents, see Supplemental Material [57].

Energy scale and sound velocity.-The CEP dispersion is given by only one energy scale, $\epsilon_{p}=\left(e^{2} / \epsilon_{0} \epsilon t_{0}\right)=$ $\left(18.1[\mathrm{eVnm}] / \epsilon t_{0}\right)$, that is determined by the size of the TBF lattice parameter $t_{0}$. For typical samples with twist angle $\theta \sim 0.01^{\circ}-0.75^{\circ}$, the corresponding lattice parameter is $t_{0} \sim 19 \mathrm{~nm}-1.4 \mu \mathrm{m}$ and the corresponding plasmonic energy scale $\epsilon_{p} \sim 2.5-200 \mathrm{meV}$ for a dielectric constant $\epsilon \approx 5$, much smaller than the bias voltage or gap of $400 \mathrm{meV}$ [47]. We thus expect a large energy window where the CEP are not damped by electron-hole excitations, especially not around the Dirac energy $E_{D} \sim \epsilon_{p} / 2$.

The plasmonic sound velocity $v_{D}$ is independent of the moiré lattice and can be approximated by $v_{D} \approx$ $3 \sqrt{3} \alpha c / \epsilon$, where $c$ is the speed of light and $\alpha \approx 1 / 137$ the fine-structure constant. In general, $v_{D} \ll c$, thus justifying our nonretarded electrostatic approach. For $\epsilon=5$, $v_{D} \sim 0.0075 c$, which is of the same order as the Fermi velocity of graphene.

Real space images of the plasmons.-A picture of the electric fields associated with the CEP can be obtained by using a scanning near field optical microscopy (SNOM) setup [65-67]. This technique consists of illuminating with an infrared laser the metallic tip of an atomic force microscope (AFM) placed on top of the TBG. The light induces an electric field dipole at the tip that oscillates with the frequency $\omega$ of the laser and this oscillation produces an electric field on the underneath TBG. In order to screen this field, the carriers of the TBG reorganize and induce collective charge excitations, that in the case of gapped TBG are CEP. These plasmons create an electric field that is backscattered in the tip and analyzing the relative variation of the scattering amplitude as a function of the position of the AFM tip it is possible to obtain real-space images of the plasmonics fields.

The tip under illumination can be modeled as an effective point dipole and the electric fields can be evaluated at $\mathbf{r}=\mathbf{r}_{0}$; see Supplemental Material [57]. We obtain real-space images of the electric near-field backscattered by the TBG's plasmons. In Figs. 4(a)-4(b), we plot the map of the amplitudes of the field in the $z$ direction, $s_{z}(\omega)$, and the corresponding phase shifts $\phi_{z}(\omega)$ for a frequency $\hbar \omega=\epsilon_{p} / 2$. Both quantities have sixfold symmetry and they are not sensitive to the chiral nature of the edge states. The brightest regions of the amplitude of $s_{z}$ correspond to the corners where the topological edges intersect. However, the phase shift of the field in the $z$ direction is strongest in the middle of the triangles of the unit cell. Similar curves are obtained for different frequencies, the main differences appear in the values of the amplitude average $\bar{s}_{z}$ and phase shift average $\bar{\phi}_{z}$ over the unit cell.
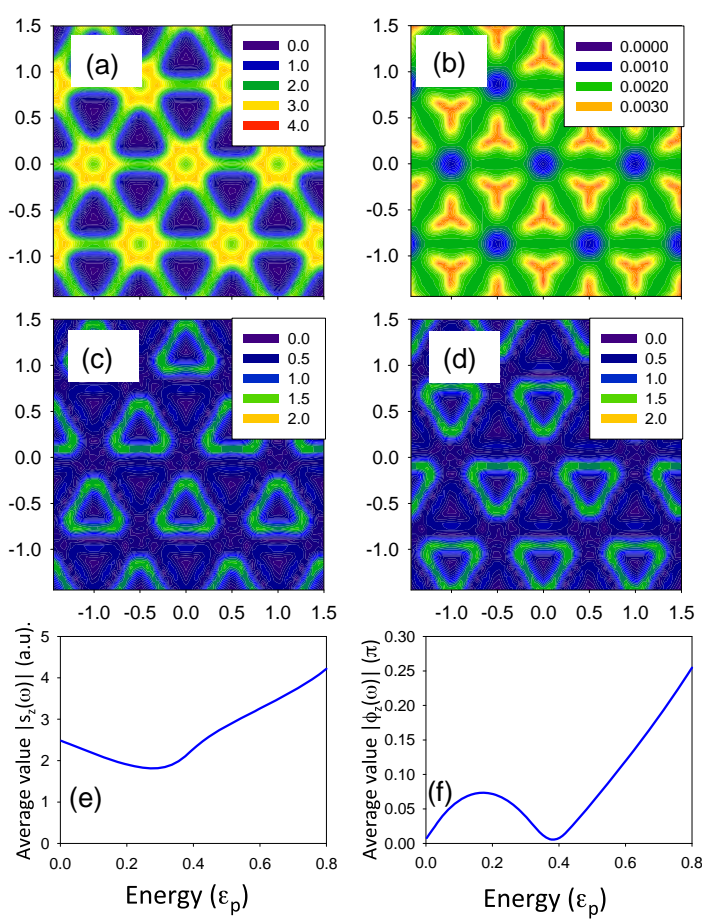

FIG. 4. Maps of the amplitude of the $z$ component (a) and phase shift (b) of the electric near field. Maps of the amplitude of the negative (c) and positive (d) circular polarized electric fields. Variation of the average amplitude (e) and phase shift (f) of the $z$ component of the near electric field as a function of the frequency. Parameters were chosen to be $z_{0}=40 \mathrm{~nm}$ and $\delta=0.005 t_{0}$.

In Figs. 4(e)-4(f), we plot these quantities as a function of the frequency. $\bar{s}_{z}$ shows a maximum at zero frequency and a minimum at energies slightly lower than the energy of the Dirac vertex $E_{D}$. However, its mathematical expression is a complicated function of plasmon frequency and little knowledge on the plasmon dispersion can be obtained. Much more information is obtained from the average value of the phase shift $\bar{\phi}_{z}$, which is proportional to the product of the frequency and the plasmon density of states. Therefore the zero phase shift at the energy $E_{D}$ indicates the absence of plasmons at this frequency and shows the existence of a Dirac cone in the plasmon dispersion.

In Figs. 4(c) and 4(d), we plot the real-space images of the amplitudes of the left, $s_{-}$, and right, $s_{+}$, circular polarized backscattered near electric fields. Because of the chirality of the edge plasmons, the electric fields generated by the plasmons have different chirality on the two triangles of the unit cell. The electric fields are located at the sides of the triangles of the unit cell. Upper triangles generate light with negative circular polarization whereas lower triangles produce positive circular polarized light.

Summary.-We have shown that the collective excitations of biased TBG when the Fermi level lies inside the gap are chiral edge plasmons that are confined at the AB-BA interfaces. Contrary to the Drude-like plasmons of Ref. [19], CEP are entirely made of intra-edge excitations 
of the topological electronic states that occur at the AB-BA edges. Because of their chiral nature, they only exist in half of the MBZ for one valley and show a large DOS at low frequencies. A simple network model shows that scattering of edge plasmons at vertices occurs maximally into the deflected directions. Most strikingly, we observe a plasmonic Dirac cone at finite energy that is composed of oscillating currents in the $x$ and $y$ directions. Breaking the rotational symmetry by a magnetic field opens up a tunable gap, paving the way towards a plasmonic transistor at tuneable frequencies $E_{D} \sim 1-100 \mathrm{meV}$ depending on the twist angle.

This work has been supported by Spain's MINECO under Grant No. PGC2018-097018-B-100, PGC2018096955-B-C42, FIS2017-82260-P, and MAT2017-88358$\mathrm{C} 3-1-\mathrm{R}$ as well as by the CSIC Research Platform on Quantum Technologies PTI-001 and the European Union Seventh Framework Programme under Grant Agreement No. 785219 and No. 881603 Graphene Flagship for Core2 and Core3. L. M. M. acknowledges Aragón Government through project Q-MAD.

[1] A. H. Castro Neto, F. Guinea, N. M. R. Peres, K. S. Novoselov, and A. K. Geim, Rev. Mod. Phys. 81, 109 (2009).

[2] M. I. Katsnelson, Graphene: Carbon in Two Dimensions (Cambridge University Press, Cambridge, England, 2012).

[3] J. M. B. Lopes dos Santos, N. M. R. Peres, and A. H. Castro Neto, Phys. Rev. Lett. 99, 256802 (2007).

[4] E. Suárez Morell, P. Vargas, L. Chico, and L. Brey, Phys. Rev. B 84, 195421 (2011).

[5] S. Shallcross, S. Sharma, E. Kandelaki, and O. A. Pankratov, Phys. Rev. B 81, 165105 (2010).

[6] E. Suárez Morell, J. D. Correa, P. Vargas, M. Pacheco, and Z. Barticevic, Phys. Rev. B 82, 121407(R) (2010).

[7] R. Bistritzer and A. H. MacDonald, Proc. Natl. Acad. Sci. U.S.A. 108, 12233 (2011).

[8] I. Brihuega, P. Mallet, H. González-Herrero, G. Trambly de Laissardière, M. M. Ugeda, L. Magaud, J. M. GómezRodriguez, F. Ynduráin, and J.-Y. Veuillen, Phys. Rev. Lett. 109, 196802 (2012).

[9] Y. Cao, V. Fatemi, S. Fang, K. Watanabe, T. Taniguchi, E. Kaxiras, and P. Jarillo-Herrero, Nature (London) 556, 43 (2018).

[10] Y. Cao, V. Fatemi, A. Demir, S. Fang, S. L. Tomarken, J. Y. Luo, J. D. Sanchez-Yamagishi, K. Watanabe, T. Taniguchi, E. Kaxiras, R. C. Ashoori, and P. Jarillo-Herrero, Nature (London) 556, 80 (2018).

[11] M. Yankowitz, S. Chen, H. Polshyn, Y. Zhang, K. Watanabe, T. Taniguchi, D. Graf, A. F. Young, and C. R. Dean, Science 363, 1059 (2019).

[12] A. L. Sharpe, E. J. Fox, A. W. Barnard, J. Finney, K. Watanabe, T. Taniguchi, M. A. Kastner, and D. GoldhaberGordon, Science 365, 605 (2019).

[13] T. Stauber, P. San-Jose, and L. Brey, New J. Phys. 15, 113050 (2013).
[14] A. Tomadin, F. Guinea, and M. Polini, Phys. Rev. B 90, 161406(R) (2014).

[15] G. X. Ni, H. Wang, J. S. Wu, Z. Fei, M. D. Goldflam, F. Keilmann, B. Ozyilmaz, A. H. Castro Neto, X. M. Xie, M. M. Fogler, and D. N. Basov, Nat. Mater. 14, 1217 (2015).

[16] T. Stauber and H. Kohler, Nano Lett. 16, 6844 (2016).

[17] F. Hu, S. R. Das, Y. Luan, T.-F. Chung, Y. P. Chen, and Z. Fei, Phys. Rev. Lett. 119, 247402 (2017).

[18] T. Stauber, T. Low, and G. Gómez-Santos, Phys. Rev. Lett. 120, 046801 (2018).

[19] S. S. Sunku, G. X. Ni, B. Y. Jiang, H. Yoo, A. Sternbach, A. S. McLeod, T. Stauber, L. Xiong, T. Taniguchi, K. Watanabe, P. Kim, M. M. Fogler, and D. N. Basov, Science 362, 1153 (2018).

[20] C. Lewandowski and L. Levitov, Proc. Natl. Acad. Sci. U.S.A. 116, 20869 (2019).

[21] N. C. H. Hesp, I. Torre, D. Rodan-Legrain, P. Novelli, Y. Cao, S. Carr, S. Fang, P. Stepanov, D. Barcons-Ruiz, H. Herzig-Sheinfux, K. Watanabe, T. Taniguchi, D. K. Efetov, E. Kaxiras, P. Jarillo-Herrero, M. Polini, and F. H. L. Koppens, arXiv:1910.07893.

[22] P. Novelli, I. Torre, F. H. L. Koppens, F. Taddei, and M. Polini, Phys. Rev. B 102, 125403 (2020).

[23] G. Sharma, M. Trushin, O. P. Sushkov, G. Vignale, and S. Adam, Phys. Rev. Research 2, 022040 (2020).

[24] E. McCann and V. I. Fal'ko, Phys. Rev. Lett. 96, 086805 (2006).

[25] I. Martin, Y. M. Blanter, and A. F. Morpurgo, Phys. Rev. Lett. 100, 036804 (2008).

[26] W. Jaskólski, M. Pelc, G. W. Bryant, L. Chico, and A. Ayuela, 2D Mater. 5, 025006 (2018).

[27] E. McCann, Phys. Rev. B 74, 161403(R) (2006).

[28] N. N. T. Nam and M. Koshino, Phys. Rev. B 96, 075311 (2017).

[29] S. G. Xu, A. I. Berdyugin, P. Kumaravadivel, F. Guinea, R. Krishna Kumar, D. A. Bandurin, S. V. Morozov, W. Kuang, B. Tsim, S. Liu, J. H. Edgar, I. V. Grigorieva, V. I. Fal'ko, M. Kim, and A. K. Geim, Nat. Commun. 10, 4008 (2019).

[30] Y.-Z. Chou, F. Wu, and S. Das Sarma, Phys. Rev. Research 2, 033271 (2020).

[31] J. Jung, F. Zhang, Z. Qiao, and A. H. MacDonald, Phys. Rev. B 84, 075418 (2011).

[32] F. Zhang, J. Jung, G. A. Fiete, Q. Niu, and A. H. MacDonald, Phys. Rev. Lett. 106, 156801 (2011).

[33] E. Prada, P. San-Jose, L. Brey, and H. Fertig, Solid State Commun. 151, 1075 (2011).

[34] A. Vaezi, Y. Liang, D. H. Ngai, L. Yang, and E.-A. Kim, Phys. Rev. X 3, 021018 (2013).

[35] F. Zhang, A. H. MacDonald, and E. J. Mele, Proc. Natl. Acad. Sci. U.S.A. 110, 10546 (2013).

[36] L. Ju, Z. Shi, N. Nair, Y. Lv, C. Jin, J. Velasco, C. OjedaAristizabal, H. A. Bechtel, M. C. Martin, A. Zettl, J. Analytis, and F. Wang, Nature (London) 520, 650 (2015).

[37] L.-J. Yin, H. Jiang, J.-B. Qiao, and L. He, Nat. Commun. 7, 11760 (2016).

[38] J. Li, K. Wang, K. J. McFaul, Z. Zern, Y. Ren, K. Watanabe, T. Taniguchi, Z. Qiao, and J. Zhu, Nat. Nanotechnol. 11, 1060 (2016).

[39] M. Kim, J.-H. Choi, S.-H. Lee, K. Watanabe, T. Taniguchi, S.-H. Jhi, and H.-J. Lee, Nat. Phys. 12, 1022 (2016). 
[40] B.-Y. Jiang, G.-X. Ni, Z. Addison, J. K. Shi, X. Liu, S. Y. F. Zhao, P. Kim, E. J. Mele, D. N. Basov, and M. M. Fogler, Nano Lett. 17, 7080 (2017).

[41] M. Kindermann, B. Uchoa, and D. L. Miller, Phys. Rev. B 86, 115415 (2012).

[42] P. San-Jose and E. Prada, Phys. Rev. B 88, 121408(R) (2013).

[43] D. K. Efimkin and A. H. MacDonald, Phys. Rev. B 98, 035404 (2018).

[44] T. Hou, Y. Ren, Y. Quan, J. Jung, W. Ren, and Z. Qiao, Phys. Rev. B 101, 201403(R) (2020).

[45] B. Tsim, N. N. T. Nam, and M. Koshino, Phys. Rev. B 101, 125409 (2020).

[46] C. De Beule, F. Dominguez, and P. Recher, Phys. Rev. Lett. 125, 096402 (2020).

[47] S. Huang, K. Kim, D. K. Efimkin, T. Lovorn, T. Taniguchi, K. Watanabe, A. H. MacDonald, E. Tutuc, and B. J. LeRoy, Phys. Rev. Lett. 121, 037702 (2018).

[48] E. H. Hasdeo and J. C. W. Song, Nano Lett. 17, 7252 (2017).

[49] S. A. Mikhailov and V. A. Volkov, J. Phys. Condens. Matter 4, 6523 (1992).

[50] V. V. Mikhailov, Zh. Eksp. Teor. Fiz. 94, 1639 (1988).

[51] V. Shikin, Pis'ma Zh. Eksp. Teor. Fiz. 47, 555 (1988).

[52] P. K. H. Sommerfeld, P. P. Steijaert, P. J. M. Peters, and R. W. van der Heijden, Phys. Rev. Lett. 74, 2559 (1995).

[53] J. C. W. Song and M. S. Rudner, Proc. Natl. Acad. Sci. U.S.A. 113, 4658 (2016).

[54] D. Jin, L. Lu, Z. Wang, C. Fang, J. D. Joannopoulos, M. Soljačić, L. Fu, and N. X. Fang, Nat. Commun. 7, 13486 (2016).

[55] A. Baldereschi and E. Tosatti, Solid State Commun. 29, 131 (1979).

[56] R. Car, E. Tosatti, S. Baroni, and S. Leelaprute, Phys. Rev. B 24, 985 (1981).
[57] See Supplemental Material at http://link.aps.org/ supplemental/10.1103/PhysRevLett.125.256804 for more details with additional analytical and numerical results which includes Refs. [58-60].

[58] M. Franco and L. Brey, Phys. Rev. Lett. 77, 1358 (1996).

[59] D. Jin, T. Christensen, M. Soljačić, N. X. Fang, L. Lu, and X. Zhang, Phys. Rev. Lett. 118, 245301 (2017).

[60] L. Brey, T. Stauber, L. Martín-Moreno, and G. GómezSantos, Phys. Rev. Lett. 124, 257401 (2020).

[61] L.-k. Shi and J. C. W. Song, Phys. Rev. X 8, 021020 (2018).

[62] In the calculation, we typically use a cutoff for the reciprocal lattice vector with $G_{\max }=40\left(2 \pi / t_{0}\right)$ that corresponds to a matrix $M_{\mathbf{G}, \mathbf{G} \text {, }}$ of dimension $4921 \times 4921$. We have checked the convergence as a function of the cutoff.

[63] J. T. Chalker and P. D. Coddington, J. Phys. C 21, 2665 (1988).

[64] H. K. Pal, S. Spitz, and M. Kindermann, Phys. Rev. Lett. 123, 186402 (2019).

[65] J. Chen, M. Badioli, P. Alonso-González, S. Thongrattanasiri, F. Huth, J. Osmond, M. Spasenović, A. Centeno, A. Pesquera, P. Godignon, A. Zurutuza Elorza, N. Camara, F. J. G. de Abajo, R. Hillenbrand, and F. H. L. Koppens, Nature (London) 487, 77 (2012).

[66] Z. Fei, A. S. Rodin, G. O. Andreev, W. Bao, A. S. McLeod, M. Wagner, L. M. Zhang, Z. Zhao, M. Thiemens, G. Dominguez, M. M. Fogler, A. H. C. Neto, C. N. Lau, F. Keilmann, and D. N. Basov, Nature (London) 487, 82 (2012).

[67] Z. Fei, A. S. Rodin, W. Gannett, S. Dai, W. Regan, M. Wagner, M. K. Liu, A. S. McLeod, G. Dominguez, M. Thiemens, A. H. Castro Neto, F. Keilmann, A. Zettl, R. Hillenbrand, M. M. Fogler, and D. N. Basov, Nat. Nanotechnol. 8, 821 (2013). 